\title{
Bonding xenon and krypton on the surface of uranium dioxide single crystal
}

Ludwik Dąbrowski, Marcin Szuta

\begin{abstract}
We present density functional theory (DFT) calculation results of krypton and xenon atoms interaction on the surface of uranium dioxide single crystal. A pseudo-potential approach in the generalised gradient approximation (GGA) was applied using the ABINIT program package. To compute the unit cell parameters, the 25 atom super-cell was chosen. It has been revealed that close to the surface of a potential well is formed for xenon and krypton atom due to its interaction with the atoms of oxygen and uranium. Depth and shape of the well is the subject of $a b$ initio calculations in adiabatic approximation. The calculations were performed both for the case of oxygenic and metallic surfaces. It has been shown that the potential well for the oxygenic surface is deeper than for the metallic surface. The thermal stability of immobilising the atoms of krypton and xenon in the potential wells were evaluated. The results are shown in graphs.
\end{abstract}

Key words: noble gases $\bullet$ uranium dioxide $\bullet$ chemical bond $\bullet$ DFT $\bullet$ GGA

L. Dąbrowski ${ }^{\bowtie}$, M. Szuta

National Centre for Nuclear Research,

7 Andrzeja Sołtana Str., 05-400 Otwock/Świerk, Poland,

Tel.: +4822 2731055 ,

E-mail: luddab@hotmail.com

Received: 17 March 2014

Accepted: 30 May 2014

\section{Introduction}

It is generally accepted that most of the insoluble inert gas atoms $\mathrm{Xe}$ and $\mathrm{Kr}$ produced during fissioning are retained in the fuel irradiated at a temperature lower than the threshold [1-10]. Some authors $[1-3,5,7,9,10]$ assume that random diffusion of gas atoms to grain boundaries and consider the effect of trapping the atoms at inter-granular bubbles until saturation occurs. To better understand the bulk Xe diffusion mechanism in uranium-based fuel, Anderson et al. [11] calculated the relevant activation energies. Others $[12,13]$ confirmed that bubbles tend to concentrate in the grain boundaries during irradiation. Likewise, some authors $[4,6,8]$ further assume that most of the gas atoms are retained in solution in the matrix of grains being there immobilised or precipitated into small fission gas bubbles.

The experimental data presented in the open literature imply that we can assume that after irradiation exposure in excess of $10^{18}$ fissions $/ \mathrm{cm}^{3}$ the single gas atom diffusion can be disregarded in description of fission gas behaviour. It means that significant fraction of fission gas products is not available for diffusion. This is a general observation for the whole temperature range of $\mathrm{UO}_{2}$ fuel that is exploited in the light water reactors (LWRs). The above well-documented assumption implies that a single gas atom diffusion model cannot be used 
to estimate the amount of fission gas that will be released from $\mathrm{UO}_{2}$ during irradiation.

In our reference [14] another approach of the issue was presented. Instead of chemical bond - the helium atom immobilisation in a deep potential well inside the crystallographic lattice was proposed. The methods estimate the energy barrier between interstitial sites in perfect lattice $\mathrm{UO}_{2}+\mathrm{He}$ on about $8 \mathrm{eV}$. We have shown there that in these circumstances the helium atom creates local bond state and performs oscillation of small amplitude, so the probability of over barrier jump to neighbour interstitial site, and hence the diffusion coefficient are close to zero.

In our work [15], in contrast to [14], in the calculations we took into account changes in local deformation during He wandering between vacancies and its impact on the barrier height, what appeared to be significant. We have shown, that over barrier jump of helium to neighbour interstitial site is associated with a high potential barrier about $4 \mathrm{eV}$ to be overcome. According to our calculations, diffusion value for the potential barrier $4.15 \mathrm{eV}$ at $300 \mathrm{~K}$ should be in the case of a perfect crystal without defects about $10^{-48} \mathrm{~cm}^{2} \cdot \mathrm{s}^{-1}$. Such a small value of diffusion coefficient effectively prevent from any helium movement in the crystals even at very high temperatures.

However, in the case of krypton the situation is different. In Ref. [16], the authors show that argon forms a weak chemical bond with uranium and oxygen. They also suggest that this should include the krypton and xenon. Xenon is heavier and has a lower ionisation potential. In our opinion it should, therefore, has a greater ability to produce chemical bonds than argon.

It is also proved that irradiating the $\mathrm{UO}_{2}$ pellets in the presence of natural xenon, part of the gas atoms is imbedded into the pellet. The xenon is found to be firmly attached to the $\mathrm{UO}_{2}$ surface such that only $1 \%$ of the attached gas can be removed after annealing samples for over $12 \mathrm{~h}$ at $1400^{\circ} \mathrm{C}$ [17].

In the work [18], we examined the ability of uranium dioxide surface to bind xenon atoms. It turned out that such a surface, in particular the oxygen surface, is able to form nearby a significant as to the depth of the potential well which can trap the xenon atoms. Because krypton in the nuclear fuel behaves similarly to xenon, we decided to check the possibilities of bonding the krypton atoms on the uranium oxide surface. Moreover, using a two-site model schema, we evaluated the values of diffusion coefficients of krypton and xenon trapped in the superficial potential wells and the thermal stability of immobilising the krypton and xenon in the potential wells at the surface.

Nuclear fuels are characterised by a total surface area depending on its density. The aim of this study is to test the ability of $\mathrm{UO}_{2}$ surface to bond the gas $\mathrm{Kr}$ and compare its ability with the Xe ability.

\section{Method of calculations}

In order to compute the ground-state properties of the system considered, an $a b$ initio plane wave pseudo-potential method base on the density functional theory (DFT) is used. We applied generally the available numerical ABINIT program package [19].

In our calculations we applied the LDA-Troullier-Martins pseudo-potential which is adapted for plane wave calculations [20]. An energy cut-off of 120 Ry was chosen.

While computing, we look for the optimal atom location that ensures a minimum value of internal energy. Each time the forces acting on the atoms (ions) are also computed. The calculations take into account the impact of eight $5 s^{2} 5 p^{6}$ electrons of xenon, six $5 f^{3} 6 d^{1} 7 s^{2}$ electrons of uranium and six $2 s^{2} 2 p^{4}$ electrons of oxygen. The remaining electrons were included into shell of cores and took into account as appropriate Troullier-Martins pseudo-potentials. The electron spin-polarisation was taken into account.

Atoms of xenon or krypton, interacting with the surface of the $\mathrm{UO}_{2}$, cause the local movement of near-surface oxygen atoms and uranium. This local nano deformation of lattice depends on the distance of the $\mathrm{Xe}$ or $\mathrm{Kr}$ from the surface. Therefore, while computing we look for the optimal atom location that ensures a minimum value of internal energy. The local lattice coordinates were computed in three stages:

Stage 1 - minimisation of the total energy through the change of lattice parameters (volume).

Stage 2 - at the obtained equilibrium lattice constants zeroing of forces acting on the separate atoms in the lattice (Hellman-Feyman forces) through the change of atom coordinates within the unit cell.

Stage 3 - iterative minimisation of the total energy through the change of lattice parameters.

\section{$\mathrm{Kr}-\mathrm{O}$ and $\mathrm{Kr}-\mathrm{U}$ interaction}

Using the above-described ABINIT software package, we in Ref. [18] received the potential dependencies of two-particles interactions for Xe-O and Xe-U, which are shown in Fig. 1. As can be seen from the calculations, the largest bonding energy for $\mathrm{Xe}-\mathrm{O}$ is $0.730 \mathrm{eV}$, and realised at the optimum distance of $1.86 \AA$, and for Xe-U respectively $-0.309 \mathrm{eV}$ at the distance of $3.06 \AA$.

Calculations carried out using the same method for the $\mathrm{Kr}-\mathrm{O}$ and $\mathrm{Kr}-\mathrm{U}$ give the bonding energy suitably -0.24 and $-0.085 \mathrm{eV}$ at optimum distances of 2.65 and $3.68 \AA$, respectively. In both cases, the interaction of the xenon with oxygen and uranium are larger than the interaction of oxygen and uranium with krypton, and the interaction with uranium in both cases are smaller than the effects with oxygen. These interactions are also presented in Fig. 1.

\section{$\mathrm{UO}_{2}$ surface}

Compound $\mathrm{UO}_{2}$ is isomorphic, with a face-centred cubic lattice (fcc) of the calcium fluorite type structure, with similar lattice parameters: $a=5.396 \AA$ [21] and space group Fm-3m (\#225).

If the plane $y z$ is accepted as the surface, then going from the surface down, we are faced with 


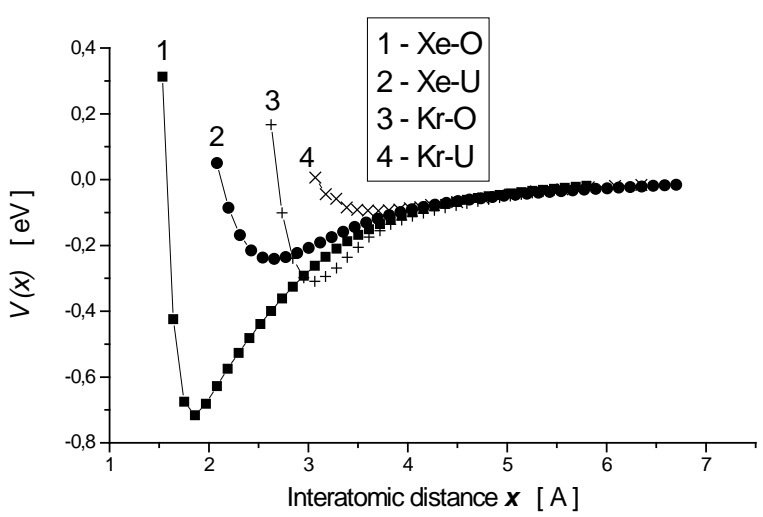

Fig. 1. Inter-atomic potential $V(x)$ for $\mathrm{Xe}-\mathrm{O}, \mathrm{Xe}-\mathrm{U}$ [from [18]], and $\mathrm{Kr}-\mathrm{O}$ and $\mathrm{Kr}-\mathrm{U}$ [this paper] vs. mutual distance of atoms.

alternating atoms of uranium, oxygen, uranium, oxygen, etc. There are therefore two possibilities: either the surface is determined by the oxygen atoms of $\mathrm{S}_{\mathrm{O}}$ (oxygenic) or uranium $\mathrm{S}_{\mathrm{U}}$ (metallic). Both of these cases were the subject of our calculations. In each of these cases, there are two extreme positions in the xenon atoms to oxygen atoms, and uranium, namely:

a) such as $\mathrm{Kr}$ atoms are located exactly opposite to the oxygen atom, and

b) when they are in front of the uranium atom.

Both of these situations are shown in Fig. 2 and refer to the configuration $\mathrm{S}_{\mathrm{O}}$. Further, these configurations will be briefly designated as $\mathrm{S}_{\mathrm{O}}, a$ and $\mathrm{S}_{\mathrm{O}}, b$, respectively.

An analogical situation exists when the surface is determined by the uranium atoms. Here, too, we have two analogous situations, which we denote as $\mathrm{S}_{\mathrm{U}}, a$ (krypton atoms are located exactly opposite to the oxygen atoms) and $\mathrm{S}_{\mathrm{U}}, b$ (krypton atoms are in front of the uranium atom), respectively. That can be

a

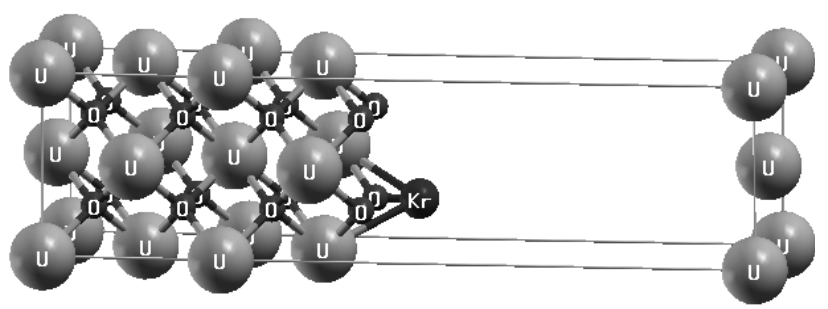

b

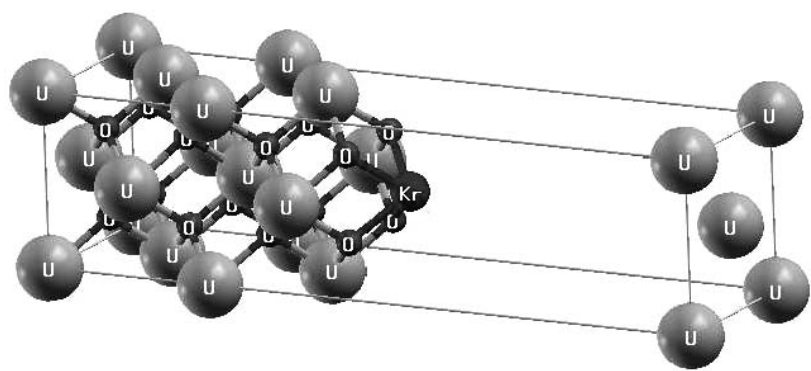

Fig. 2. Bonding of krypton on the surface of uranium dioxide in the position over an oxygen atom. (a) $\mathrm{S}_{\mathrm{O}}, a$ configuration. (b) $\mathrm{S}_{\mathrm{o}}, b$ configuration. easily imagined, if in Figs. 2a and 2b krypton atoms are located on the left side of shown super-cells.

\section{Potential well}

For numerical calculations we have chosen 25 atomic super-cell of dimensions $4 a \times a \times a$ (21.88 $\times 5.47 \times 5.47 \AA$ ), which we filled half of it with 8 uranium atoms and 16 oxygen atoms. The second half of the super-cell is a space free from oxygen and the uranium atoms. In this space we placed the krypton atom in positions $\mathrm{S}_{\mathrm{O}}, a, \mathrm{~S}_{\mathrm{O}}, b, \mathrm{~S}_{\mathrm{U}}, a$ and $\mathrm{S}_{\mathrm{U}}, b$. Because the kryptonon atom is separated from the nearest oxygen atoms or uranium atoms by the distance of nearly two lattice constants (see Fig. 2), we assume that $\mathrm{Kr}$ interacts really only with $\mathrm{U}$ and $\mathrm{O}$ atoms, located on the left side, it means with the atoms of the surface and subsurface layers. It does not interact with the atoms located on the right side, and belonging to the next, periodically repeated super-cell. Binding energy depending on the distance between $\mathrm{Kr}$ and $\mathrm{UO}_{2}$ surface, and thus the shape and depth of the potential well, in which the krypton atom is close to the surface, for the configuration of $\mathrm{S}_{\mathrm{O}}, a, \mathrm{~S}_{\mathrm{o}}, b$, are shown in Fig. 3a, and for the configuration of $\mathrm{S}_{\mathrm{U}}, a$ and $\mathrm{S}_{\mathrm{U}}, b$ in Fig. $3 \mathrm{~b}$.

For comparison, in Figs. $3 \mathrm{a}$ and $3 \mathrm{~b}$ also is shown that the oxygenic surface, bonds more strongly the krypton and xenon atoms than the surface of uranium metal, what is shown in Table 1 . Given the fact that the oxygen-xenon bonding is more than two times higher than uranium-krypton and ura-
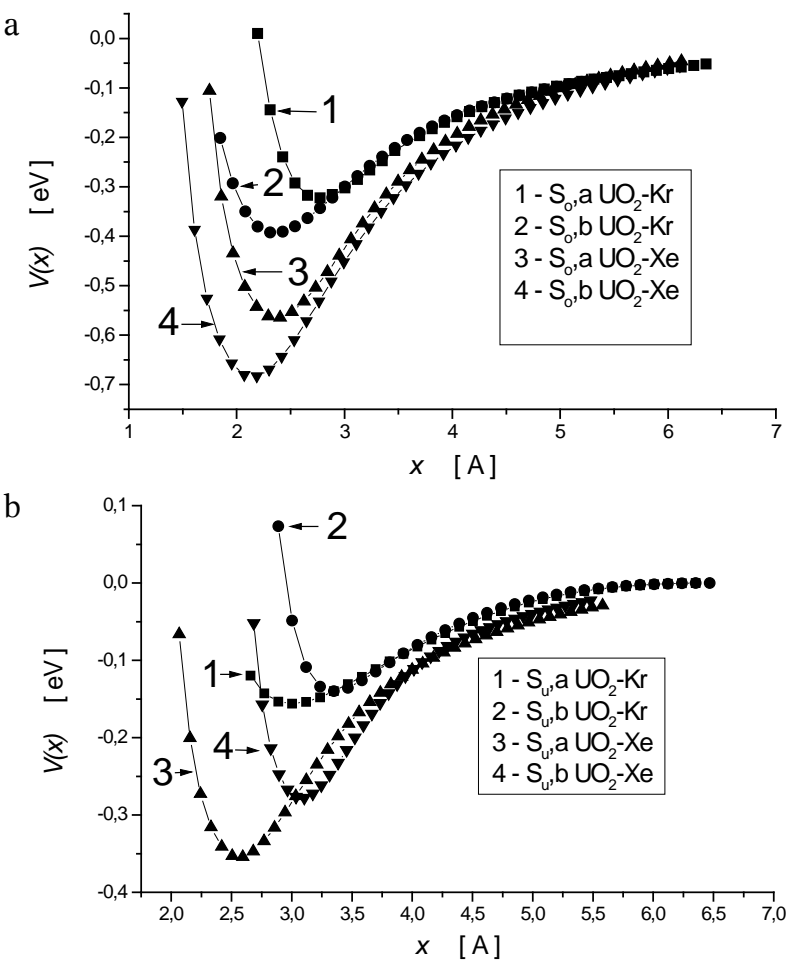

Fig. 3. (a) The bonding energy depending on $x$ in the $\mathrm{S}_{\mathrm{O}}$ configurations $x-\mathrm{Kr}$ or Xe distance - from the surface of $\mathrm{UO}_{2}$. (b). The bonding energy depending on $x$ in the $\mathrm{S}_{\mathrm{U}}$ configurations: $x-\mathrm{Kr}$ or Xe distance - from the surface of $\mathrm{UO}_{2}$. 
Table 1. Summary results of the well depth $\mathrm{U}_{0}$ and the optimal distance from the $\mathrm{X}_{\mathrm{opt}}$ surface for various configurations for $\mathrm{Kr}$ and $\mathrm{Xe}$

\begin{tabular}{lcccccccc}
\hline \multicolumn{1}{c}{ Configuration } & $\mathrm{S}_{\mathrm{O}}, a \mathrm{Kr}$ & $\mathrm{S}_{\mathrm{o}}, b \mathrm{Kr}$ & $\mathrm{S}_{\mathrm{U}}, a \mathrm{Kr}$ & $\mathrm{S}_{\mathrm{U}}, b \mathrm{Kr}$ & $\mathrm{S}_{\mathrm{O}}, a \mathrm{Xe}$ & $\mathrm{S}_{\mathrm{O}}, b \mathrm{Xe}$ & $\mathrm{S}_{\mathrm{U}}, a \mathrm{Xe}$ & $\mathrm{S}_{\mathrm{U}}, b \mathrm{Xe}$ \\
\hline $\mathrm{X}_{\mathrm{opt}}[\AA]$ & 2.77 & 2.35 & 3.00 & 3.36 & 2.37 & 2.15 & 2.56 & 3.08 \\
$\mathrm{U}_{0}[\mathrm{eV}]$ & 0.32 & 0.39 & 0.16 & 0.14 & 0.57 & 0.68 & 0.35 & 0.28 \\
\hline
\end{tabular}

nium-xenon bonding, and that are realised on the shorter distances - energetically more favourable configuration of $S_{O}$ does not seem to be a surprise. This is in full correlation with the values of the inter-atom potentials $\mathrm{Kr}-\mathrm{O}, \mathrm{Kr}-\mathrm{U}, \mathrm{Xe}-\mathrm{O}$ and $\mathrm{Xe}-\mathrm{U}$ as presented in Fig. 1.

Summary of the bonding energy of all configurations and the optimum distance from the surface are shown in Table 1.

As can be seen from Table 1, from the four configurations of the krypton and four configurations of the xenon, two of them, namely $\mathrm{S}_{\mathrm{O}}, b$, from the oxygenic surface and $\mathrm{S}_{\mathrm{U}}, a$, from the metallic side, have the lowest energy and they will be referred in the process of bonding. Only these two configurations, as preferred, will be dealt with in later work.

\section{Diffusion of krypton and xenon}

\section{Diffusion perpendicular to the surface}

Let us assume that the atom of krypton or xenon is located between two oxygenic surfaces in the potential well, disposed nearby one of the surface. Then, after some time, it jumps along the $x$-axis, perpendicular to the surface to the next analogous well, being nearby the second surface. This model is shown in Fig. 4. The analogous situation occurs on the uranium (metallic) surface.

In our previous works $[14,15]$ in order to solve the problems related to the diffusion of helium in $\mathrm{UO}_{2}$, we used harmonic oscillator approach. However, due to the peculiar shape of potential well, which is poorly approximated by the parabola (see Figs. 3a and 3b), the WKB approximation (see Appendix) appeared to be better. The results of numeric calculations of diffusion coefficients are presented in Fig. 5.

\section{Diffusion parallel to the surface}

Atoms of krypton or xenon, being found in the local minimums of the potential of the crystalline field, marked in the Table 1 as the positions $\mathrm{S}_{\mathrm{O}}, b-\mathrm{UO}_{2}-\mathrm{Kr}$,

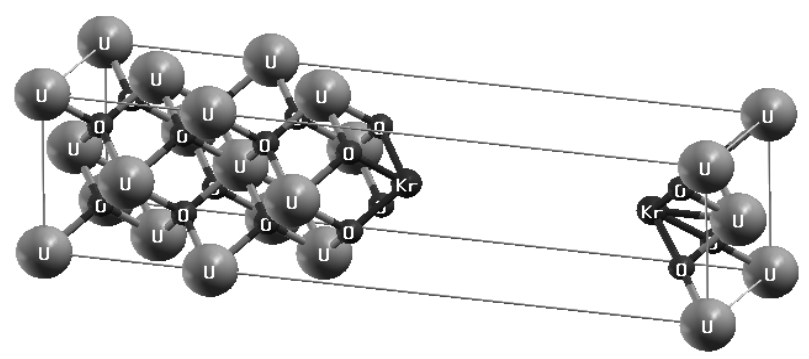

Fig. 4. Two oxygenic surfaces of $\mathrm{UO}_{2}$ between which takes place the migration.

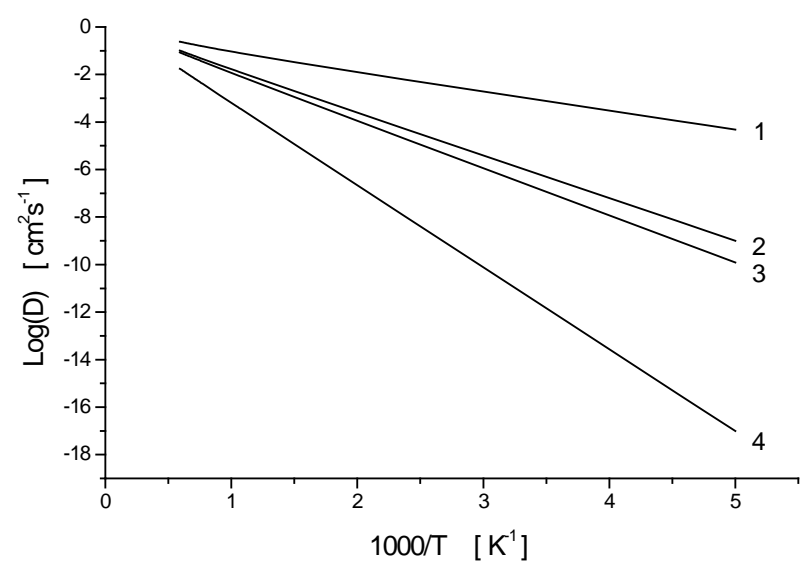

Fig. 5. Temperature dependence of perpendicular diffusion coefficients for krypton and xenon depending from configurations: 1,3 - configurations $\mathrm{S}_{\mathrm{U}}, a$ and $\mathrm{S}_{\mathrm{O}}, b$ for krypton; 2,4 - configurations $\mathrm{S}_{\mathrm{U}}, a$ and $\mathrm{S}_{\mathrm{O}}, b$ for xenon.

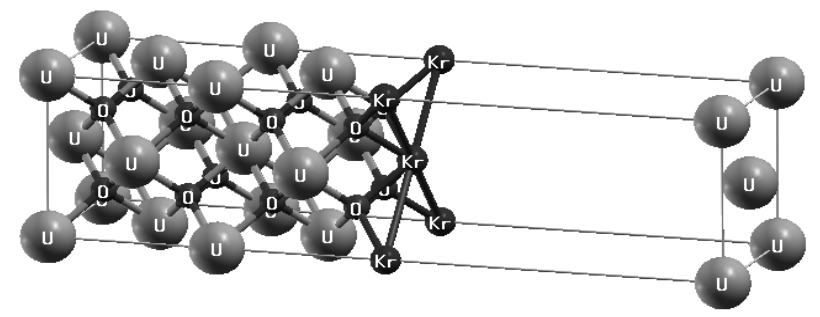

Fig. 6. Crystalline equivalent positions of krypton (xenon) atoms on the oxygenic surface $\mathrm{UO}_{2}$, which can be occupied by the atoms during diffusion.

$\mathrm{S}_{\mathrm{O}}, b-\mathrm{UO}_{2}-\mathrm{Xe}, \mathrm{S}_{\mathrm{U}}, a-\mathrm{UO}_{2}-\mathrm{Kr}$ and $\mathrm{S}_{\mathrm{U}}, a-\mathrm{UO}_{2}-\mathrm{Xe}$, can jump over to other, equivalent crystallographic positions. And so, for example, the atom of krypton or xenon, occupying the position $\mathrm{S}_{\mathrm{O}}, b$ on the oxygenic surface, can jump to neighbouring position $\mathrm{S}_{\mathrm{O}}, b$ overcoming small local potential barrier in the position $\mathrm{S}_{\mathrm{O}}, a$. This is illustrated in Fig. 6.

The position $\mathrm{S}_{\mathrm{O}}, a$ is the saddle point - shows the local minimum in the perpendicular direction to the surface and the local maximum in the parallel direction. As this result from the values, specified in the Table 1 , in this case the potential height of the barrier for xenon atom is $0.12 \mathrm{eV}$ and for krypton atom $0.07 \mathrm{eV}$. Similarly occurs the situation on the uranium (metallic) surface with the difference that the local minimum of energy is in the $\mathrm{S}_{\mathrm{U}}, a$ position, and the saddle point is in the $\mathrm{S}_{\mathrm{U}}, b$ position. Potential barriers in this case for xenon is $0.07 \mathrm{eV}$ and for krypton $0.016 \mathrm{eV}$. The above quoted values of potential barriers show that adsorption of the noble gases of xenon and argon on the $\mathrm{UO}_{2}$ surface is the localised adsorption.

Applying the WKB method (see Appendix) the results of numeric calculations are presented in Fig. 7. 


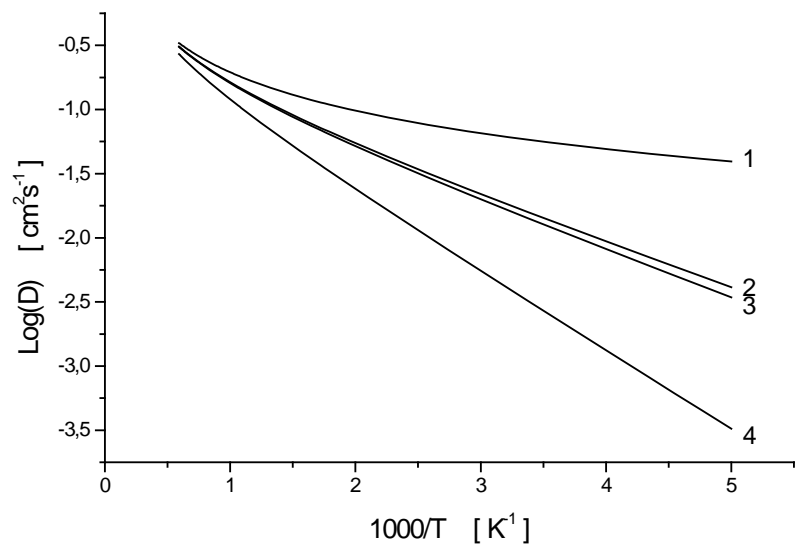

Fig. 7. Temperature dependence of surface diffusion coefficients for krypton and xenon depending on surfaces: 1 , 3 - uranium (metallic) and oxygenic surfaces for krypton; 2, 4- uranium (metallic) and oxygenic surfaces for xenon.

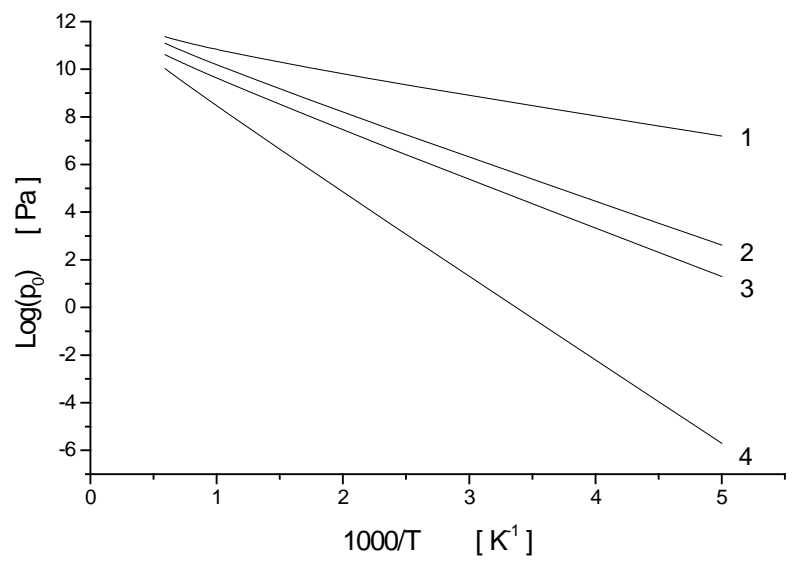

Fig. 8. Dependence of equilibrium gas pressure on temperature from configurations: 1,3 - configurations $\mathrm{S}_{\mathrm{U}}, a$ and $\mathrm{S}_{\mathrm{O}}, b$ for krypton; 2,4 - configurations $\mathrm{S}_{\mathrm{U}}, a$ and $\mathrm{S}_{\mathrm{O}}, b$ for xenon.

\section{Escape of krypton and xenon atoms from the potential well}

If the distance between the planes, schematically shown in Fig. 4 will be greater than the mean free path of particles in the gas surrounding the $\mathrm{UO}_{2}$ surface, then, as a result of collisions of the atoms, our krypton or xenon atoms have difficult access to the opposite surface. Let us assume that in the surrounding gas there are also particles of escaping krypton (xenon) atoms. The situation is similar to the evaporation of the liquid. At the proper pressure, stream of particles escaping away from the surface will be compensated with the stream of particles inflowing from the gas. Applying the WKB method (see Appendix), the results of numerical computations of this pressure are shown in Fig. 8. As far as the temperature rises, like the pressure of saturated steam in the case of liquid, this pressure grows up. When this pressure is equal to atmospheric - the violent escape of krypton (xenon) atoms follows from the surface, what would be equivalent to the boiling point in the case of liquid. This temperature for the oxygenic surface is equal to $510 \mathrm{~K}$ for the xenon and $314 \mathrm{~K}$ for the krypton. For the uranium (metallic) surface, these temperatures are 269 and $130 \mathrm{~K}$, respectively.

\section{Discussion}

Defined by us the inter-atomic potential interactions of krypton and xenon with oxygen and uranium show that bonds with xenon are twice stronger than with krypton. On the other hand bonds of these noble gases with uranium are about two times weaker than with oxygen. This is reflected in the interaction potentials with the surface of $\mathrm{UO}_{2}$. The oxygenic surface produces the potential well for xenon atoms with a depth of $0.68 \mathrm{eV}$, and for the krypton atoms with a depth of $0.39 \mathrm{eV}$. Meanwhile the metallic (uranium) surface - only $0.35 \mathrm{eV}$ for Xe and $0.16 \mathrm{eV}$ for $\mathrm{Kr}$. These interactions are weak in comparison with the chemical bonds of other elements. They are not able to immobilise xenon and krypton on the surface in the shape of a chemical compound. The situation reminds rather the behaviour of the liquid. When the temperature of surroundings is low - the process runs slowly and the escape of the atoms of noble gases from the surface can be compensated with the stream of the same particles being in the gas surrounding the $\mathrm{UO}_{2}$ surface. The situation undergoes the essential change, when the effective pressure of noble gas from the side of the surface crosses the value of the atmospheric pressure. The process can no longer be in equilibrium, similarly as while boiling of the liquid. It should be noticed that this process can run in the opposite side - cooling the system we can bring again to condensation of $\mathrm{Xe}$ or $\mathrm{Kr}$ on the $\mathrm{UO}_{2}$ surface. The analogy with liquid is all the more justified, that heat of vaporisation, e.g. $\mathrm{H}_{2} \mathrm{O}$ per molecule is approximately $0.42 \mathrm{eV}$. Assuming that the heat of vaporisation and the height of potential barrier of water have the similar values we can expect that only xenon on the oxygen surface will have the 'boiling' temperature higher than water, and the remaining configuration - lower. These expectations, as it results from the above quoted numeric values in paragraph "Escape of krypton and xenon atoms from the potential well", are completely confirmed. The received values of 'boiling' temperature for Xe and $\mathrm{Kr}$ suggest that such a mechanism of immobilising of considered here noble gases can have the significant influence on the content of these gases in the fuel. This relates, in particular, to the narrow slots of a width of less than $620 \AA$ (mezoslots), which surfaces can very long exchange that atoms of xenon or krypton.

\section{Conclusion}

The noble gases krypton and xenon have a significant chemical activity in relation to oxygen and uranium, and especially to oxygen. This causes that on the $\mathrm{UO}_{2}$ surface arise the potential wells of significant depth.

Adsorption of $\mathrm{Xe}$ and $\mathrm{Kr}$ on the surface of $\mathrm{UO}_{2}$ occurs in the form of a mono atomic layer as localised and can be observed mainly in low temperatures below room temperature. The exception is oxygenic surface for which the 'boiling' temperature is equal to about $510 \mathrm{~K}$ for xenon can significantly contribute to immobilisation of these gas in the fuel. 
Behaviour of theses gases near the $\mathrm{UO}_{2}$ surface reminds the process of evaporation of the liquid. The gas $\mathrm{Kr}$ or Xe 'evaporating' from the surface produces definite pressure, increasing with temperature. At a given 'boiling' temperature and above that temperature, follows rapid release of atoms of these gases from the surface.

The nano narrow cracks in the $\mathrm{UO}_{2}$, which thickness does not exceed the free path of particles in the surrounding atmosphere can be effective traps for fission gas products.

\section{References}

1. Bellamy, R. G., \& Rich, J. B. (1969). Grain boundary gas release and swelling in high burn-up uranium dioxide. J. Nucl. Mater., 33(1), 64-76.

2. Hargreaves, R., \& Collins, D. M. (1976). A quantitative model for fission gas release and swelling in irradiated uranium dioxide. J. Br. Nucl. Energy Soc., 15, 311-318.

3. Ito, K., Iwasaki, R., \& Yoshihiko, I. (1985). Finite element model of fission gas release from $\mathrm{UO}_{2}$ fuel. J. Nucl. Sci. Technol., 22(2), 129-138.

4. Mac Iwan, J. R., \& Stevens, W. H. (1964). Xenon diffusion in $\mathrm{UO}_{2}$. J. Nucl. Mater., 11(1), 77-93.

5. Nakajima, T., \& Saito, H. (1987). A comparison between fission gas release data and FEMAXI-IV. Nucl. Eng. Des., 101(3), 267-279.

6. Ray, I. L. F., Thiele, H., \& Matzke, H. (1992). Transmission electron microscopy study of fission product behaviour in high burnup $\mathrm{UO}_{2}$. J. Nucl. Mater., 188, 90-95.

7. Rest, J., \& Cronenberg, W. A. (1987). Modeling the behavior of Xe, I, Cs, Te, Ba and Sr in solid and liquefied fuel during severe accidents. J. Nucl. Mater., 150(2), 203-225.

8. Szuta, M. (1994). The diffusion coefficient of fission-product rare gases in single crystal uranium dioxide during irradiation. J. Nucl. Mater., 210, 178-186.

9. White, R. J., \& Tucker, M. O. (1983). A new fission-gas release model. J. Nucl. Mater., 118, 1-38.

\section{Appendix}

Application of Wentzel, Kramers, Brillouin (WKB) approximation to solution the problems relevant to diffusion xenon and krypton near the $\mathrm{UO}_{2}$ surface.

\section{Appendix I. Penetration through the potential barrier}

This method allows to obtain approximate solution to the time-independent Schrödinger equation and particularly useful in calculating tunnelling rates through potential barriers. The wave function is expressed as the exponential of a function:

$$
\Psi(x)=e^{\Phi(x)}
$$$$
\text { where } \quad \Phi^{\prime \prime}(x)+\Phi^{\prime}(x)^{2}=\frac{2 m}{\hbar^{2}}(V(x)-E)
$$

Such transformation of the wave function allows obtaining approximate solutions of Schrödinger
10. Zimmermann, H. (1978). Investigations on swelling and fission gas behaviour in uranium dioxide. J. Nucl. Mater., 75, 154-161.

11. Anderson, D. A., Uberuaga, B. P. P., \& Nericar, N. (2011). U and Xe transport in $\mathrm{UO}_{2 \pm \mathrm{x}}$ : Density functional theory calculations. Phys. Rev. B, 84, 054105-17.

12. Burbach, J., \& Zimmermann, H. (1985). Spaltgasverhalten in bestrahltem $\mathrm{UO}_{2}$ bei out-of-pile-Gluehungen von 1400 bis $2000^{\circ} \mathrm{C}$. $K f K, 3997,1-39$.

13. Une, K., \& Kashibe, S. (1990). Fission gas release during post irradiation annealing of BWR fuels. $J$. Nucl. Sci. Technol., 27, 1002-1016.

14. Dąbrowski, L., \& Szuta, M. (2012). Ab initio study of helium atoms immobilization in $\mathrm{UO}_{2}$ crystals. Nukleonika, 57(3), 337-343.

15. Dabrowski, L., \& Szuta, M. (2013). Diffusion of helium in the perfect uranium and thorium dioxide single crystals. Nukleonika, 58(2), 295-300.

16. Li, J., Liang, B. E., \& Andrews, L. (2002). Noble gas-actinide compounds: complexation of the CUO molecule by $\mathrm{Ar}, \mathrm{Kr}$ and $\mathrm{Xe}$ atoms in noble gas matrices. Science, 295(22), 2242-2245.

17. Turnbull, J. A. (1980). Review of irradiation induced resolution in oxide fuels. Radiat. Eff., 53(3/4), 243-249.

18. Dąbrowski, L., \& Szuta, M. (2013). Bonding xenon on the surface of uranium dioxide single crystal. Nukleonika, 58(4), 453-458.

19. www.abinit.org.

20. Troullier, N., \& Martins, J. L. (1991). Efficient pseudopotentials for plane - wave calculations. Phys. Rev. B, 43(3), 1993-2006.

21. Beauvy, M. (1992). Nonideality of the solid solution in (U,Pu) $\mathrm{O}_{2}$ nuclear fuels. J. Nucl. Mater., 188, 232-238.

22. Razavy, M. (2003). Quantum theory of tunneling. River Edge: World Scientific Publishing.

23. Feynmann, R. P., Leighton, R. B., \& Sands, M. L. (1974). The Feynmann lectures on physics. (Vol. 3, pp. 125-130). Warsaw: PWN.

24. Grabert, H., \& Schober, H. R. (1997). Theory of tunneling and diffusion of light interstitial in metals. In H. Wipf (Ed.) Hydrogen in metals III: Properties and applications (pp. 5-49). Topics in applied physics. Vol. 73. Berlin: Springer.

equation in the form:

$$
\Psi(x) \approx C \frac{e^{i \int d x \sqrt{\frac{2 m}{\hbar^{2}}(E-V(x))+\theta}}}{\sqrt[4]{\frac{2 m}{\hbar^{2}}(E-V(x))}}
$$

in the area, where the energy of the particle is larger than the height of the potential barrier, and

$$
\Psi(x) \approx \frac{C_{+} e^{\int d x \sqrt{\frac{2 m}{\hbar^{2}}(V(x)-E)}}+C_{-} e^{-\int d x \sqrt{\frac{2 m}{\hbar^{2}}(V(x)-E)}}}{\sqrt[4]{\frac{2 m}{\hbar^{2}}(E-V(x))}}
$$

in the opposite case.

Omitting technical details [22] the transmission coefficient for a particle tunnelling through a single potential barrier is: 


$$
T=\frac{e^{-2 \int_{x 1}^{x 2} d x \sqrt{\frac{2 m}{\hbar^{2}}(V(x)-E)}}}{\left\{1+\frac{1}{4} e^{-2 \int_{x 1}^{x 2} d x \sqrt{\frac{2 m}{\hbar^{2}}(V(x)-E)}}\right\}^{2}}
$$

For particles of larger energies than the barrier heights the $T$ coefficient is equal to the unity.

Appendix 2. The model of diffusion perpendicular to the surface for the potential of the crystalline field presented schematically in Fig. app.

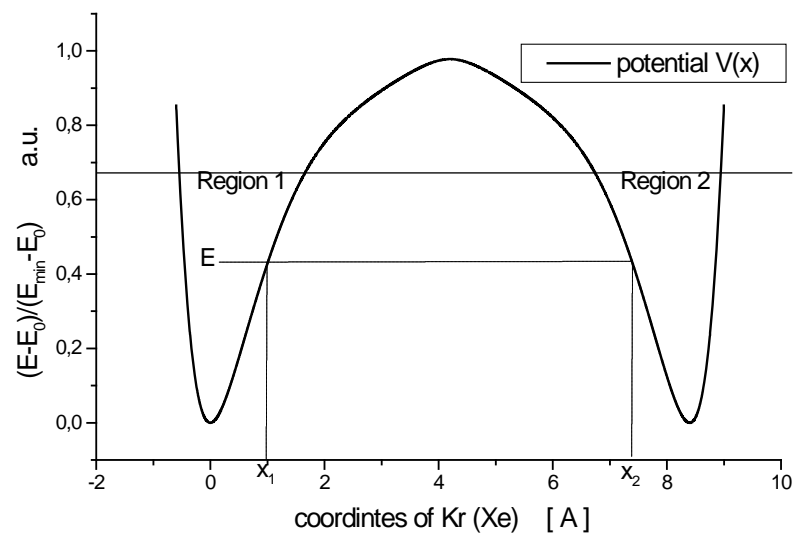

Fig. app. Two-site model schema.

\section{Appendix 2.1. Penetration through the potential barrier}

The model of diffusion perpendicular to the surface for the potential of the crystalline field presented schematically in Fig. app.

The particle (Kr, Xe) with energy $E$, being in the potential well in region 1 , in the consequence of the tunnel effect, penetrates to region 2 partly. If the particle in the region 1 has an energy $E$, The total energy of particles, which find themselves in region 2 is:

$$
\begin{aligned}
H_{12} & =\int_{0}^{V / k_{B} T} p\left(E / k_{B} T\right) T(E) E d\left(E / k_{B} T\right) \\
& +\int_{V / k_{B} T}^{\infty} p\left(E / k_{B} T\right) E d\left(E / k_{B} T\right)
\end{aligned}
$$

where $p$ is the probability of finding a particle of energy $E$.

If we place the particle in the well in region $2-$ the situation will look identically: the particle will penetrate to area 1 from area 2 due to tunnel effect. It is obvious that $H_{12}=H_{21}$. This is the typical example of the two-site Hamiltonian. In such case, if in the moment $t=0$ the particle was observed in the region 1 , then this is the unstable situation. If we mark by $C_{1}$ state of the amplitude of the particle being in area 1 , and by $C_{2}$ state of the amplitude of the particle in area 2, then according to the general principals of quantum mechanics, the amplitude of $C_{i}$ states, which previously were constants, begin to depend on time. This is described by the linear system of differential equations [23]:

$$
\begin{aligned}
& i \hbar \frac{d C_{1}}{d t}=H_{11} C_{1}+H_{12} C_{2} \\
& i \hbar \frac{d C_{2}}{d t}=H_{21} C_{1}+H_{22} C_{2}
\end{aligned}
$$

Omitting the technical details we get finally:

$$
C_{1}(t)=\exp \left(-\frac{i H_{11} t}{\hbar}\right) \cos \left(\frac{H_{12} t}{\hbar}\right)
$$

The amplitude $C_{1}$ decreases with time as the cosine function and reaches the value zero after $\tau=$ $\left(\pi \hbar / 2 H_{12}\right)$ while $C_{2}$ increases as sine function and reaches the value 1 for the same time $T$. In other words the $\mathrm{Kr}(\mathrm{Xe})$ atom moved from the region 1 to region 2 after time $T$. The rate $v=1 / \tau$ also determines the quantum diffusion coefficient $D$ [24]:

$$
D=\frac{z}{6}(2 d)^{2} v
$$

where $z$ is the number of neighbouring interstitial sites and $2 d$ is the distance between two sites.

Such process of diffusion occurs, when the distance on which the particles diffuse is smaller than the free path of $\mathrm{Xe}$ or $\mathrm{Kr}$ atoms in the surrounding gas. It is equal approximately $621 \AA$ in the air in normal conditions. This is approx. 74 times more than the distance between wells in Fig. app.

\section{Appendix 2.2. The conditions of equilibrium between the 'evaporating' surface $\mathrm{UO}_{2}$ and the surrounding atmosphere}

As it results from Eq. (A8), on average after the time $\tau=\left(\pi \hbar / 2 H_{12}\right)$ the particle will leave the potential well. The total stream of particles leaving the well during the unit time is:

$$
I_{-}=\frac{n}{d_{0}^{2}} T=\frac{2 n H_{12}}{\pi d_{0}^{2} \hbar}
$$

where $n / d_{0}^{2}$ - surface density of absorbed particles by the surface $\mathrm{UO}_{2}$.

From the other side, using the kinetic-molecular theory of gases, for the stream of particles in the opposite direction, it means from the side of gas towards the surface we will receive:

$$
I_{+}=\frac{1}{6} p \sqrt{\frac{2}{m k_{B} T}}
$$

where $p$ - gas pressure, $m$ - particle mass of Xe or $\mathrm{Kr}, T$ - absolute temperature.

If $I_{-}=I_{+}$, then though the escape of atoms exists from the area of the well, then it is compensated by the stream of particles, being in the gas. The equilibrium pressure $p_{0}$ is:

$$
p_{0}=\frac{12 n H_{12}}{\pi d_{0}^{2} \hbar} \sqrt{\frac{m k_{B} T}{2}}
$$

where $p_{0}$ is the saturated vapour pressure in equilibrium at a given temperature. 